Research Article

\title{
Fixed Points of Integral Type Multivalued Contractive Mappings with $w$-Distance
}

\author{
Haiyan Gao (D), $^{1,2}$ Haoyue Wang, ${ }^{3}$ Lili Zhang, ${ }^{4}$ and Liangshi Zhao ${ }^{5}{ }^{5}$ \\ ${ }^{1}$ School of Mathematics, Dongbei University of Finance and Economics, Dalian, Liaoning 116025, China \\ ${ }^{2}$ Department of General Studies, Dalian University of Finance and Economics, Dalian, Liaoning 116000, China \\ ${ }^{3}$ Department of Mathematics, Liaoning Normal University, Dalian, Liaoning 116029, China \\ ${ }^{4}$ Department of General Studies, Dalian Neusoft University of Information, Dalian, Liaoning 116023, China \\ ${ }^{5}$ Center for Studies of Marine Economy and Sustainable Development, Liaoning Normal University, Dalian, \\ Liaoning 116029, China
}

Correspondence should be addressed to Liangshi Zhao; liangshizhao85@163.com

Received 4 March 2020; Revised 19 May 2020; Accepted 23 May 2020; Published 16 June 2020

Academic Editor: Kishin Sadarangani

Copyright (c) 2020 Haiyan Gao et al. This is an open access article distributed under the Creative Commons Attribution License, which permits unrestricted use, distribution, and reproduction in any medium, provided the original work is properly cited.

Two fixed point theorems for multivalued contractive mappings involving $w$-distance and inequalities of integral type are proved. Our results generalize a few results in the literature. Two illustrative examples are given.

\section{Introduction and Preliminaries}

In 2002, Branciari [1] generalized the famous Banach contraction principle and proved the following fixed point theorem for the contractive mapping of the integral type.

Theorem 1 (see [1]). Let $T$ be a mapping from a complete metric space $(X, d)$ into itself satisfying

$$
\int_{0}^{\mathrm{d}(T x, T y)} \varphi(t) \mathrm{d} t \leq c \int_{0}^{\mathrm{d}(x, y)} \varphi(t) \mathrm{d} t, \quad \forall x, y \in X,
$$

where $c \in(0,1)$ is a constant and $\varphi: \mathbb{R}^{+} \longrightarrow \mathbb{R}^{+}$is Lebesgue integrable, summable in each compact subset of $\mathbb{R}^{+}$and $\int_{0}^{\varepsilon} \phi(t) d t>0$ for each $\varepsilon>0$. Then, $T$ has a unique fixed point $a \in X$ and $\lim _{n \longrightarrow \infty} T^{n} x=a$ for each $x \in X$.

Using Hausdorff metric, Nadler [2] introduced the concept of multivalued contraction and proved a multivalued version of the well-known Banach contraction principle.

Theorem 2 (see [2]). Let $(X, d)$ be a complete metric space and let $T$ be a mapping from $X$ into $C B(X)$, where $C B(X)$ is the family of all nonempty closed and bounded subsets of $X$. Assume that there exists $c \in[0,1)$ such that

$$
H(T(x), T(y)) \leq c \mathrm{~d}(x, y), \quad \forall x, y \in X .
$$

Then, $T$ has a fixed point.

In the past decades, various fixed point theorems concerning multivalued contractive mappings have been proved. Especially, Feng and Liu [3] generalized Theorem 2 and proved a few fixed point theorems for multivalued contractive mappings without Hausdorff metric.

Theorem 3 (see [3]). Let $(X, d)$ be a complete metric space and $T$ be a multivalued mapping from $X$ into $C L(X)$, where $C L(X)$ is the family of all nonempty closed subsets of $X$. Assume that

$\left(a_{1}\right)$ There exist constants $b, c \in(0,1)$ with $c<b$ such that, for any $x \in X$, there is $y \in T(x)$ satisfying

$$
\begin{aligned}
b \mathrm{~d}(x, y) & \leq \mathrm{d}(x, T(x)), \\
\mathrm{d}(y, T(y)) & \leq c \mathrm{~d}(x, y) .
\end{aligned}
$$

$\left(a_{2}\right) \quad f: X \longrightarrow \mathbb{R}^{+}$is lower semicontinuous, where $f(x)=d(x, T(x)), \forall x \in X$.

Then, $T$ has a fixed point in $X$. 
In 1996, Kada et al. [4] introduced the concept of $w$-distance in a metric space and proved several fixed point theorems for single-valued contractive mappings under $w$-distance. Some other fixed point results concerning $w$-distance can be found in [5-9]. In 2007, Guran [5] deduced the following fixed point theorem, which is a generalization of Theorem 3 .

Theorem 4 (see [5]). Let $(X, d)$ be a complete metric space, $T: X \longrightarrow C L(X)$ be a multivalued mapping, $w: X \times X \longrightarrow \mathbb{R}^{+}$be a $w$-distance on $X$, and $b \in(0,1)$. Assume that

$\left(b_{1}\right)$ There exists $c \in(0,1)$, with $c<b$, such that, for any $x \in X$, there is $y \in T(x)$ satisfying

$\Phi_{1}=\left\{\phi \mid \phi: \mathbb{R}^{+} \longrightarrow \mathbb{R}^{+}\right.$is Lebesgue integrable and summable in each compact subset of $\mathbb{R}^{+}$and $\left.\int_{0}^{\mathcal{E}} \phi(t) \mathrm{d} t>0, \forall \varepsilon>0\right\}$

$\Phi_{2}=\left\{\phi \mid \phi\right.$ belongs to $\Phi_{1}$ and satisfies that $\left.\int_{0}^{a+b} \phi(t) \mathrm{d} t \leq \int_{0}^{a} \phi(t) \mathrm{d} t+\int_{0}^{b} \phi(t) \mathrm{d} t, \forall a, b \in \mathbb{R}^{+}\right\}$.

Definition 1 (see [4]). Let $(X, d)$ be a metric space. A function $w: X \times X \longrightarrow \mathbb{R}^{+}$is called a $w$-distance in $X$ if it satisfies the following:

$\left(w_{1}\right) w(x, z) \leq w(x, y)+w(y, z), \forall x, y, z \in X$

$\left(w_{2}\right)$ For each $x \in X$, a mapping $w(x, \cdot): X \longrightarrow \mathbb{R}^{+}$is lower semicontinuous

$\left(w_{3}\right)$ For any $\varepsilon>0$, there exists $\delta>0$ such that $w(z, x) \leq \delta$ and $w(z, y) \leq \delta$ imply $\mathrm{d}(x, y) \leq \varepsilon$

Example 1. Let $X$ be a normed linear space with norm $\|\cdot\|, \alpha$ be a positive constant, and $w: X \times X \longrightarrow \mathbb{R}^{+}$be defined by

$$
w(x, y)=\|y\|^{\alpha}, \quad \forall x, y \in X .
$$

Then, $w$ is a $w$-distance in $X$.

Proof. Let $x, y, z \in X$. It is clear that $\left(w_{2}\right)$ holds and

$$
w(x, z)=\|z\|^{\alpha} \leq\|y\|^{\alpha}+\|z\|^{\alpha}=w(x, y)+w(y, z),
$$

which implies $\left(w_{1}\right)$. For each $\varepsilon>0$, put $\delta=(\varepsilon / 2)^{\alpha}$. If $w(z, x) \leq \delta$ and $w(z, y) \leq \delta$, it follows that

$$
\begin{aligned}
\mathrm{d}(x, y)= & \|x-y\| \leq\|x\|+\|y\|=w^{1 / \alpha}(z, x)+w^{1 / \alpha}(z, y) \\
& \leq \delta^{1 / \alpha}+\delta^{1 / \alpha}=\varepsilon,
\end{aligned}
$$

which yields $\left(w_{3}\right)$. That is, $w$ is a $w$-distance in $X$.

Example 2. Let $X=\mathbb{R}$ be endowed with the Euclidean metric $d=|\cdot|$ and $w: X \times X \longrightarrow \mathbb{R}^{+}$be defined by

$$
\begin{aligned}
b w(x, y) & \leq w(x, T(x)), \\
w(y, T(y)) & \leq c w(x, y) .
\end{aligned}
$$

$\left(b_{2}\right) \quad f_{w}: X \longrightarrow \mathbb{R}^{+}$is lower semicontinuous, where $f_{w}(x)=w(x, T(x)), \forall x \in X$.

Then, $T$ has a fixed point in $X$.

Motivated by the results in [1, 3-5], we prove two fixed point results for multivalued contractive mappings of integral type with respect to $w$-distance in complete metric spaces. The results presented in this paper improve Theorems 2-4. Two examples with uncountably many points are included.

Throughout this paper, we denote by $\mathbb{N}$ the set of positive integers, $\mathbb{N}_{0}=\{0\} \cup \mathbb{N}, \mathbb{R}=(-\infty,+\infty), \mathbb{R}^{+}=[0,+\infty)$, and

$$
w(x, y)=\max \{|a||x-y|,|a x-y|\}, \quad \forall x, y \in X,
$$

where $a$ is a constant in $\mathbb{R}$. Then, $w$ is a $w$-distance in $X$.

Proof. Let $x, y, z \in X$. It is clear that $\left(w_{2}\right)$ holds and

$$
\begin{aligned}
w(x, z) & =\max \{|a||x-z|,|a x-z|\} \\
& \leq \max \{|a||x-y|+|a||y-z|,|a||x-y|+|a y-z|\} \\
& \leq \max \{|a||x-y|,|a x-y|\}+\max \{|a||y-z|,|a y-z|\} \\
& =w(x, y)+w(y, z),
\end{aligned}
$$

which implies $\left(w_{1}\right)$. For each $\varepsilon>0$, put $\delta=(\varepsilon / 2)$. If $w(z, x) \leq \delta$ and $w(z, y) \leq \delta$, it is easy to see that

$$
\begin{aligned}
\mathrm{d}(x, y) & =|x-y| \leq|a z-x|+|a z-y| \\
& \leq \max \{|a||z-x|,|a z-x|\}+\max \{|a||z-y|,|a z-y|\} \\
& =w(z, x)+w(z, y) \\
& \leq \delta+\delta \\
& =\varepsilon,
\end{aligned}
$$

which yields $\left(w_{3}\right)$. That is, $w$ is a $w$-distance in $X$.

Let $(X, d)$ be a metric space. For any $u \in X, D \subseteq X$, $T: X \longrightarrow \mathrm{CL}(X)$, and $w: X \times X \longrightarrow \mathbb{R}^{+}$, put

$$
\begin{aligned}
\mathrm{d}(u, D) & =\inf _{y \in D} \mathrm{~d}(u, y), \\
w(u, D) & =\inf _{y \in D} w(u, y), \\
f(u) & =\mathrm{d}(u, T(u)), \\
f_{w}(u) & =w(u, T(u)) .
\end{aligned}
$$


A sequence $\left\{x_{n}\right\}_{n \in \mathbb{N}_{0}}$ in $X$ is called an orbit of $T$ if $x_{n} \in T\left(x_{n-1}\right)$ for all $n \in \mathbb{N}$.

Definition 2. Let $(X, d)$ be a metric space and $T: X \longrightarrow \mathrm{CL}(X)$ be a multivalued mapping. A function $g: X \longrightarrow \mathbb{R}^{+}$is said to be

$\left(a_{1}\right)$ Lower semicontinuous in $X$ if $g(y) \leq \liminf _{n \longrightarrow \infty} g\left(y_{n}\right)$ for each $y \in X$ and $\left\{y_{n}\right\}_{n \in \mathbb{N}_{0}} \subseteq X$ with $\lim _{n \longrightarrow \infty} y_{n}=y$

$\left(a_{2}\right) T$-orbitally lower semicontinuous at $z \in X$ if $g(z) \leq \liminf _{n \longrightarrow \infty} g\left(x_{n}\right)$ for each orbit $\left\{x_{n}\right\}_{n \in \mathbb{N}_{0}}$ of $T$ with $\lim _{n \longrightarrow \infty} x_{n}=z$

$\left(a_{3}\right)$ T-orbitally lower semicontinuous in $X$ if it is $T$-orbitally lower semicontinuous at each $z \in X$

Obviously, if $g$ is lower semicontinuous in $X$, then $g$ is $T$-orbitally lower semicontinuous in $X$.

The following lemmas play important roles in this paper.

Lemma 1 (see [10]). Let $\varphi \in \Phi_{1}$ and $\left\{r_{n}\right\}_{n \in \mathbb{N}}$ be a nonnegative sequence. Then, $\lim _{n \longrightarrow \infty} \int_{0}^{r_{n}} \varphi(t) d t=0$ if and only if $\lim _{n \rightarrow \infty} r_{n}=0$.

Lemma 2 (see [4]). Let $X$ be a metric space with metric $d$ and let $w$ be a $w$-distance in $X$. Let $\left\{x_{n}\right\}_{n \in \mathbb{N}}$ and $\left(y_{n}\right)_{n \in \mathbb{N}}$ be sequences in $X$, let $\left(\alpha_{n}\right)_{n \in \mathbb{N}}$ and $\left\{\beta_{n}\right\}_{n \in \mathbb{N}}$ be sequences in $\mathbb{R}^{+}$ converging to 0 , and let $x, y, z \in X$, then the following hold:

(a) If $w\left(x_{n}, y\right) \leq \alpha_{n}$ and $w\left(x_{n}, z\right) \leq \beta_{n}$ for any $n \in \mathbb{N}$, then $y=z$. In particular, if $w(x, y)$ and $w(x, z)=0$, then $y=z$.

(b) If $w\left(x_{n}, y_{n}\right) \leq \alpha_{n}$ and $w\left(x_{n}, z\right) \leq \beta_{n}$ for any $n \in \mathbb{N}$, then $\left\{y_{n}\right\}_{n \in \mathbb{N}}$ converges to $z$.

(c) If $w\left(x_{n}, x_{m}\right) \leq \alpha_{n}$ for any $n, m \in \mathbb{N}$ with $n>m$, then $\left\{x_{n}\right\}_{n \in \mathbb{N}}$ is a Cauchy sequence.

(d) If $w\left(x, x_{n}\right) \leq \alpha_{n}$ for any $n \in \mathbb{N}$, then $\left\{x_{n}\right\}_{n \in \mathbb{N}}$ is a Cauchy sequence.

Lemma 3 (see [11]). Let $(X, d)$ be a metric space, $w$ be a $w$-distance on $X$, and $D \in C L(X)$. Suppose that there exists $u \in X$ such that $w(u, u)=0$. Then, $w(u, D)=0$ if and only if $u \in D$.

\section{Fixed Point Theorems}

In this section, we establish fixed point theorems for multivalued contractive mappings (13) and (21), respectively.

Theorem 5. Let $(X, d)$ be a complete metric space, $w$ be a $w$-distance in $X$, and $T: X \longrightarrow C L(X)$ satisfy that for each $x \in X$ there is $y \in T(x)$ with

$$
\begin{gathered}
b \int_{0}^{w(x, y)} \varphi(t) \mathrm{d} t \leq \int_{0}^{w(x, T(x))} \varphi(t) \mathrm{d} t, \\
\int_{0}^{w(y, T(y))} \varphi(t) \mathrm{d} t \leq c \int_{0}^{w(x, y)} \varphi(t) \mathrm{d} t,
\end{gathered}
$$

where $b$ and $c$ are constants in $(0,1)$ with $c<b$ and $\varphi \in \Phi_{2}$. Then,

$\left(c_{1}\right)$ For each $x_{0} \in X$, there exists an orbit $\left\{x_{n}\right\}_{n \in \mathbb{N}_{0}}$ of $T$ such that $\lim _{n \longrightarrow \infty} x_{n}=u$ for some $u \in X$.

$\left(c_{2}\right) f_{w}(u)=0$ if $f_{w}: X \longrightarrow \mathbb{R}^{+}$is $T$-orbitally lower semicontinuous at $u$. Moreover, $u$ is a fixed point of $T$ if $w(u, u)=0$.

Proof. Now, we show $\left(c_{1}\right)$. Let $x_{0}$ be an arbitrary point in $X$ and $a=(c / b)$. It follows from (13) that there exists $x_{1} \in T\left(x_{0}\right)$ such that

$$
\begin{gathered}
b \int_{0}^{w\left(x_{0}, x_{1}\right)} \varphi(t) \mathrm{d} t \leq \int_{0}^{w\left(x_{0}, T\left(x_{0}\right)\right)} \varphi(t) \mathrm{d} t, \\
\int_{0}^{w\left(x_{1}, T\left(x_{1}\right)\right)} \varphi(t) \mathrm{d} t \leq c \int_{0}^{w\left(x_{0}, x_{1}\right)} \varphi(t) \mathrm{d} t .
\end{gathered}
$$

Continuing this process, we choose easily a sequence $\left\{x_{n}\right\}_{n \in \mathbb{N}_{0}}$ in $X$ satisfying

$$
\begin{gathered}
x_{n+1} \in T\left(x_{n}\right), \\
b \int_{0}^{w\left(x_{n}, x_{n+1}\right)} \varphi(t) \mathrm{d} t \leq \int_{0}^{w\left(x_{n}, T\left(x_{n}\right)\right)} \varphi(t) \mathrm{d} t, \quad \forall n \in \mathbb{N}_{0}, \\
\int_{0}^{w\left(x_{n+1}, T\left(x_{n+1}\right)\right)} \varphi(t) \mathrm{d} t \leq c \int_{0}^{w\left(x_{n}, x_{n+1}\right)} \varphi(t) \mathrm{d} t, \quad \forall n \in \mathbb{N}_{0} .
\end{gathered}
$$

Next, we claim that $\left\{x_{n}\right\}_{n \in \mathbb{N}_{0}}$ is a Cauchy sequence. It follows from (15) and $\varphi \in \Phi_{2}$ that

$$
\begin{aligned}
& 0 \leq \int_{0}^{w\left(x_{n}, x_{n+1}\right)} \varphi(t) \mathrm{d} t \leq a \int_{0}^{w\left(x_{n-1}, x_{n}\right)} \varphi(t) \mathrm{d} t \leq a^{n} \int_{0}^{w\left(x_{0}, x_{1}\right)} \varphi(t) \mathrm{d} t, \quad \forall n \in \mathbb{N}_{0}, \\
& 0 \leq \int_{0}^{w\left(x_{n}, T\left(x_{n}\right)\right)} \varphi(t) \mathrm{d} t \leq a \int_{0}^{w\left(x_{n-1}, T\left(x_{n-1}\right)\right)} \varphi(t) \mathrm{d} t \leq a^{n} \int_{0}^{w\left(x_{0}, T\left(x_{0}\right)\right)} \varphi(t) \mathrm{d} t, \quad \forall n \in \mathbb{N}_{0} .
\end{aligned}
$$
that

Letting $n \longrightarrow \infty$ in (16) and using Lemma 1 , we infer

$$
\begin{array}{r}
\lim _{n \longrightarrow \infty} w\left(x_{n}, x_{n+1}\right)=0, \\
\lim _{n \longrightarrow \infty} f_{w}\left(x_{n}\right)=0 .
\end{array}
$$


Making use of (16), $\left(w_{1}\right)$ and $\varphi \in \Phi_{2}$, we conclude that

$$
\begin{aligned}
\int_{0}^{w\left(x_{n}, x_{m}\right)} \varphi(t) \mathrm{d} t & \leq \int_{0}^{\sum_{i=n}^{m-1} w\left(x_{i}, x_{i+1}\right)} \varphi(t) \mathrm{d} t \leq \sum_{i=n}^{m-1} \int_{0}^{w\left(x_{i}, x_{i+1}\right)} \varphi(t) \mathrm{d} t \leq \sum_{i=n}^{m-1} a^{i} \int_{0}^{w\left(x_{0}, x_{1}\right)} \varphi(t) \mathrm{d} t \\
& \leq \frac{a^{n}}{1-a} \int_{0}^{w\left(x_{0}, x_{1}\right)} \varphi(t) \mathrm{d} t, \quad \forall m, n \in \mathbb{N} \text { with } m>n,
\end{aligned}
$$

which together with Lemmas 1 and 2 yields that $\left\{x_{n}\right\}_{n \in \mathbb{N}_{0}}$ is a Cauchy sequence. Completeness of $X$ implies that there exists some $u \in X$ such that

$$
\lim _{n \longrightarrow \infty} x_{n}=u \text {. }
$$

Finally, we show $\left(c_{2}\right)$. Since $f_{w}: X \longrightarrow \mathbb{R}^{+}$is $T$-orbitally lower semicontinuous at $u$, it follows from (17) that

$$
0 \leq f_{w}(u) \leq \liminf _{n \longrightarrow \infty} f_{w}\left(x_{n}\right)=0,
$$

which means that $f_{w}(u)=0$. Thus, Lemma 3 and $w(u, u)=$ 0 yield that $u \in T(u)$. This completes the proof.

Theorem 6. Let $(X, d)$ be a complete metric space, $w$ be a $w$-distance in $X$, and $T: X \longrightarrow C L(X)$ satisfy that for each $(x, y) \in X \times T(x)$, and there exists $z \in T(y)$ with

$$
\int_{0}^{w(y, z)} \varphi(t) \mathrm{d} t \leq c \int_{0}^{w(x, y)} \varphi(t) \mathrm{d} t
$$

where $c$ is a constant in $(0,1)$ and $\varphi \in \Phi_{2}$. Then, $\left(c_{1}\right)$ and $\left(c_{2}\right)$ hold.

Proof. Now, we show $\left(c_{1}\right)$. Let $\left(x_{0}, x_{1}\right)$ be an arbitrary point in $X \times T\left(x_{0}\right)$. It follows from (21) that there exists $x_{2} \in T\left(x_{1}\right)$ such that

$$
\int_{0}^{w\left(x_{1}, x_{2}\right)} \varphi(t) \mathrm{d} t \leq c \int_{0}^{w\left(x_{0}, x_{1}\right)} \varphi(t) \mathrm{d} t .
$$

Continuing this process, we construct a sequence $\left\{x_{n}\right\}_{n \in \mathbb{N}_{0}}$ in $X$ satisfying

$$
\begin{aligned}
& x_{n+1} \in T\left(x_{n}\right), \quad \forall n \in \mathbb{N}_{0}, \\
& \int_{0}^{w\left(x_{n}, x_{n+1}\right)} \varphi(t) \mathrm{d} t \leq c \int_{0}^{w\left(x_{n-1}, x_{n}\right)} \varphi(t) \mathrm{d} t, \quad \forall n \in \mathbb{N} .
\end{aligned}
$$

Next, we claim that $\left\{x_{n}\right\}_{n \in \mathbb{N}_{0}}$ is a Cauchy sequence. It follows from (23) and $\varphi \in \Phi_{2}$ that

$$
0 \leq \int_{0}^{w\left(x_{n}, x_{n+1}\right)} \varphi(t) \mathrm{d} t \leq c^{n} \int_{0}^{w\left(x_{0}, x_{1}\right)} \varphi(t) \mathrm{d} t, \quad \forall n \in \mathbb{N} .
$$

Letting $n \longrightarrow \infty$ in (24) and using Lemma 1, we infer that

$$
\lim _{n \longrightarrow \infty} w\left(x_{n}, x_{n+1}\right)=0,
$$

which together with (23) implies that

$$
0 \leq f_{w}\left(x_{n}\right) \leq w\left(x_{n}, x_{n+1}\right), \quad \forall n \in \mathbb{N} .
$$

Combining (25) and (26), we conclude that

$$
\lim _{n \longrightarrow \infty} f_{w}\left(x_{n}\right)=0 .
$$

The rest of the proof is similar to that of Theorem 5 and is omitted. This completes the proof.

Remark 1. Theorem 5 generalizes Theorems 2-4. The below example demonstrates that Theorem 5 generalizes indeed Theorems 2 and 3.

Example 3. Let $X=[0,(5 / 4)]$ be endowed with the $\mathrm{Eu}$ clidean metric $d=|\cdot|, \quad u=0, \quad w: X \times X \longrightarrow \mathbb{R}^{+}$, $T: X \longrightarrow \mathrm{CL}(X)$, and $\varphi: \mathbb{R}^{+} \longrightarrow \mathbb{R}^{+}$be defined by

$$
w(x, y)=y, \quad \forall x, y \in X,
$$

$$
\begin{aligned}
T(x) & =\left\{\begin{aligned}
\left\{\frac{3}{4} x^{2}\right\}, & \forall x \in[0,1) \cup\left(1, \frac{5}{4}\right], \\
\left\{\frac{3}{4}, \frac{4}{5}\right\}, & x=1,
\end{aligned}\right. \\
c & =\frac{15}{17} \\
b & =\frac{16}{17}, \\
\varphi(t) & =1, \quad \forall t \in \mathbb{R}^{+} .
\end{aligned}
$$

It is clear that $w(u, u)=0, \varphi \in \Phi_{2}$, and

$$
f_{w}(x)=w(x, T(x))=\frac{3}{4} x^{2}, \quad \forall x \in X,
$$

is continuous in $X$. It follows that $f_{w}(x)$ is lower semicontinuous in $X$. In order to verify (13), for each $x \in X$, we have to consider the following cases.

Case 1. $x \in[0,1) \cup(1,(2 / \sqrt{3})) \cup((2 / \sqrt{3}),(5 / 4)]$. Clearly, there exists $y=(3 / 4) x^{2} \in T(x)=\left\{(3 / 4) x^{2}\right\}$ satisfying

$$
\begin{gathered}
b \int_{0}^{w(x, y)} \varphi(t) \mathrm{d} t=\frac{16}{17} \cdot \frac{3}{4} x^{2} \leq \frac{3}{4} x^{2}=\int_{0}^{w(x, T(x))} \varphi(t) \mathrm{d} t, \\
\int_{0}^{w(y, T(y))} \varphi(t) \mathrm{d} t=\frac{27}{64} x^{4} \leq \frac{15}{17} \cdot \frac{3}{4} x^{2}=c \int_{0}^{w(x, y)} \varphi(t) \mathrm{d} t .
\end{gathered}
$$


Case 2. $x=1$. It is clear that there exists $y=(3 / 4) \in T(x)=$ $\{(3 / 4),(4 / 5)\}$ such that

$$
\begin{gathered}
b \int_{0}^{w(x, y)} \varphi(t) \mathrm{d} t=\frac{16}{17} \cdot \frac{3}{4} \leq \frac{3}{4}=\int_{0}^{w(x, T(x))} \varphi(t) \mathrm{d} t, \\
\int_{0}^{w(y, T(y))} \varphi(t) \mathrm{d} t=\frac{27}{64} \leq \frac{15}{17} \cdot \frac{3}{4}=c \int_{0}^{w(x, y)} \varphi(t) \mathrm{d} t .
\end{gathered}
$$

Case 3. $x=(2 / \sqrt{3})$. It follows that there exists $y=1 \in T(x)=\left\{(3 / 4) x^{2}\right\}$ such that

$$
\begin{gathered}
b \int_{0}^{w(x, y)} \varphi(t) \mathrm{d} t=\frac{16}{17} \cdot 1 \leq 1=\int_{0}^{w(x, T(x))} \varphi(t) \mathrm{d} t, \\
\int_{0}^{w(y, T(y))} \varphi(t) \mathrm{d} t=\frac{3}{4} \leq \frac{15}{17} \cdot 1=c \int_{0}^{w(x, y)} \varphi(t) \mathrm{d} t .
\end{gathered}
$$

Hence, (13) holds. That is, the conditions of Theorem 5 are satisfied. It follows from Theorem 5 that $T$ has a fixed point $0 \in X$.

However, we cannot invoke Theorem 3 to show that the mapping $T$ has a fixed point in $X$. Suppose that the conditions of Theorem 3 are satisfied. Take $x_{*}=1$. For $y_{*} \in T x_{*}=\{(3 / 4),(4 / 5)\}$, we consider two possible cases as follows:

Case 1: $y_{*}=(3 / 4)$. It follows that

$$
\frac{21}{64}=\mathrm{d}\left(\frac{3}{4}, \frac{27}{64}\right)=\mathrm{d}\left(y_{*}, T\left(y_{*}\right)\right) \leq c \mathrm{~d}\left(x_{*}, y_{*}\right)=\frac{1}{4} c,
$$

which implies that $c \geq(21 / 16)>1$, which is impossible because $c \in(0,1)$.

Case 2: $y_{*}=(4 / 5)$. It is easy to see that

$$
\frac{8}{25}=\mathrm{d}\left(\frac{4}{5}, \frac{12}{25}\right)=\mathrm{d}\left(y_{*}, T\left(y_{*}\right)\right) \leq c \mathrm{~d}\left(x_{*}, y_{*}\right)=\frac{1}{5} c,
$$

which implies that $c \geq(8 / 5)>1$, which is a contradiction because $c \in(0,1)$.

It follows from Remark 1 in [3] that Theorem 3 extends Theorem 2. Thus, Theorem 2 is not applicable in proving the existence of fixed points for the multivalued contractive mapping $T$ in $X$.

Remark 2. The following example shows that Theorem 6 is different from Theorem 2 .

Example 4. Let $X=\mathbb{R}^{+}$be endowed with the Euclidean metric $d=|\cdot|, w: X \times X \longrightarrow \mathbb{R}^{+}, T: X \longrightarrow \mathrm{CL}(X)$, and $\varphi: \mathbb{R}^{+} \longrightarrow \mathbb{R}^{+}$be defined by

$$
\begin{aligned}
w(x, y) & =y, \quad \forall x, y \in X, \\
T(x) & =[0, x], \quad \forall x \in X \\
\varphi(t) & =t^{-(1 / 2)}, \quad \forall t \in \mathbb{R}^{+} .
\end{aligned}
$$

Put $c \in((1 / 12), 1)$. Clearly, $\varphi \in \Phi_{2}, w(0,0)=0,(X, d)$ is a complete metric space, and

$$
f_{w}(x)=w(x, T(x))=\inf \{t: t \in[0, x]\}=0, \quad \forall x \in X,
$$

is continuous in $X$.

For each $(x, y) \in X \times T(x)$, there exists $z=(y / 4) \in T(y)$ with

$$
\int_{0}^{w(y, z)} \varphi(t) \mathrm{d} t=\sqrt{y} \leq 2 c \sqrt{y}=c \int_{0}^{w(x, y)} \varphi(t) \mathrm{d} t,
$$

that is, (21) holds. It follows from Theorem 6 that $T$ has a fixed point $0 \in X$.

However, Theorem 2 is useless in proving the existence of fixed points for the mapping $T$ in $X$. Suppose that (2) holds. Take $x_{*}=1$ and $y_{*}=2$. It follows that

$$
H\left(T\left(x_{*}\right), T\left(y_{*}\right)\right)=H(T(1), T(2))=1 \leq c=c \mathrm{~d}(1,2)=c \mathrm{~d}\left(x_{*}, y_{*}\right) \text {, }
$$

which is impossible because $c \in[0,1)$.

\section{Data Availability}

The data used to support the findings of this study are included within the article.

\section{Conflicts of Interest}

The authors declare that they have no conflicts of interest.

\section{Authors' Contributions}

All authors contributed equally to the writing of this paper. All authors read and approved the final manuscript.

\section{Acknowledgments}

This work was supported by the National Natural Science Foundation of China (no. 41701616).

\section{References}

[1] A. Branciari, "A fixed point theorem for mappings satisfying a general contractive con of integral type," International Journal of Mathematics and Mathematical Sciences, vol. 29, no. 9, pp. 531-536, 2002.

[2] S. Nadler Jr., "Multi-valued contraction mappings," Pacific Journal of Mathematics, vol. 30, no. 2, pp. 475-488, 1969.

[3] Y. Feng and S. Liu, "Fixed point theorems for multi-valued contractive mappings and multi-valued Caristi type mappings," Journal of Mathematical Analysis and Applications, vol. 317, no. 1, pp. 103-112, 2006.

[4] O. Kada, T. Suzuki, and W. Takahashi, "Nonconvex minimization theorems and fixed point theorems in complete metric spaces," Mathematica Japonica, vol. 44, pp. 381-391, 1996.

[5] L. Guran, "Fixed points for multivalued operators with respect to a $w$-distance on metric spaces," Carpathian Journal of Mathematics, vol. 23, pp. 89-92, 2007. 
[6] M. Imdad and F. Rouzkard, "Fixed point theorems in ordered metric spaces via $w$-distances," Fixed Point Theory and Applications, vol. 2012, no. 1, p. 17, 2012.

[7] H. Lakzian, H. Aydi, and B. E. Rhoades, "Fixed points for $(\phi, \psi, p)$-weakly contractive mappings in metric spaces with $w$-distance," Applied Mathematics and Computation, vol. 219, no. 12, pp. 6777-6782, 2013

[8] H. Lakzian, V. Rakočević, and H. Aydi, "Extensions of Kannan contraction via $w$-distances," Aequationes Mathematicae, vol. 93, no. 6, pp. 1231-1244, 2019.

[9] R. Zuhra, M. S. M. Noorani, and F. Shaddad, "Contraction mapping principle in partially ordered quasi metric space concerning to $w$-distances," The Journal of Nonlinear Sciences and Applications, vol. 10, no. 2, pp. 699-712, 2017.

[10] Z. Liu, X. Li, S. M. Kang, and S. Y. Cho, "Fixed point theorems for mappings satisfying contractive conditions of integral type and applications," Fixed Point Theory and Applications, vol. 2011, no. 1, p. 18, 2011.

[11] L.-J. Lin and W.-S. Du, "Some equivalent formulations of the generalized Ekeland's variational principle and their applications," Nonlinear Analysis: Theory, Methods \& Applications, vol. 67, no. 1, pp. 187-199, 2007. 